\title{
GREEN'S FUNCTIONS FOR INTERIOR AND EXTERIOR HELMHOLTZ PROBLEMS
}

\author{
Stanistaw Kukla, Urszula Siedlecka, Izabela Zamorska \\ Institute of Mathematics, Czestochowa University of Technology, Poland \\ stanislaw.kukla@im.pcz.pl,urszula.siedlecka@im.pcz.pl,izabela.zamorska@im.pcz.pl
}

\begin{abstract}
In the paper, the derivation of Green's functions for Helmholtz equation in circular, annular and exterior circular domains is presented. The Green's functions are assumed of the form a cosine series. An example of application of the Green's functions in frequency analysis of a composite membrane is presented.
\end{abstract}

\section{Introduction}

The Green's functions were successfully applied to solving various differential problems formulated in mathematical modeling of physical processes [1-3]. Particularly, the class of problems describing with Helmholtz equation can be solved by using the Green's function method. The first stage in application of the method is deriving the necessary Green's functions.

Derivations of the Green's functions for various differential equations are presented in many monographs and articles [1-3]. The Green's functions for the twodimensional the Helmholtz equation over rectangular domain with Dirichlet and Neumann boundary conditions are given in the book [1]. Also the Green's functions for Helmholtz equation when the domain consists of the annular rings with Dirichlet conditions or Neumann conditions along the inner and outer boundaries are presented in this book. Unfortunately, the Green's functions over annular rings for other combination of Dirichlet and Neumann conditions at inner and outer boundaries are not given. These Green's functions are needed for deriving a solution to the vibration problem of a composite circular and annular membrane consisting with annular rings, each of constant density $[4,5]$. In the paper [3] the Green's function for the Helmholtz differential equation with an impedance boundary condition is discussed.

In this paper the derivation of the Green's functions for interior Helmholtz equations and free space Green's functions for exterior Helmholtz equations is presented.

The Green's functions for the Helmholtz equation defined over the plane (free space Green's functions) are applied to finding an approximate solution of Helmholtz problem by using the fundamental solution method $[6,7]$. We consider here 
the domain $D=R^{2} \backslash K$, where $K$ is a circle. The free space Green's functions in two cases are presented: the Green's functions which satisfies Dirichlet or Neumann conditions at the boundary $\partial K$.

\section{Green's functions for interior Helmholtz problems}

Let us consider the Helmholtz equation in a circular or an annular domain $D$

$$
\frac{\partial^{2} G_{H}}{\partial r^{2}}+\frac{1}{r} \frac{\partial G_{H}}{\partial r}+\frac{1}{r^{2}} \frac{\partial^{2} G_{H}}{\partial \theta^{2}}+\lambda^{2} G_{H}=\frac{\delta(r-\zeta) \delta\left(\theta-\theta^{\prime}\right)}{r},(r, \theta) \in D
$$

The solution of this equation can be obtained by using the method of separation of variables. In our consideration we assume function $G_{H}$ in the form:

$$
G_{H}\left(r, \theta, \zeta, \theta^{\prime}\right)=\sum_{n=-\infty}^{\infty} G_{n}(r, \xi) \cos n\left(\theta-\theta^{\prime}\right)
$$

This leads to differential equation and boundary conditions respect to variable $r$. Green's function $G_{n}(r, \zeta)$ for this boundary problem satisfies a non-homogeneous equation

$$
\frac{1}{r} \frac{d}{d r}\left(r \frac{d G_{n}(r, \zeta)}{d r}\right)+\left(\lambda^{2}-\frac{n^{2}}{r^{2}}\right) G_{n}(r, \zeta)=\frac{-\delta(r-\zeta)}{2 \pi r}
$$

and the homogeneous boundary conditions. We consider equation (3) in circular or annular domains. We assume Dirichlet or Neumann boundary conditions.

The solution of this equation can be presented in the form [7]

$$
G_{n}(r, \zeta)=g_{0 n}(r, \zeta)+g_{1 n}(r, \zeta) H(r-\zeta)
$$

The function $g_{0 n}$ is the general solution of the homogeneous equation, which is obtained from equation (3) by replacing of the right-hand side with the zero function. The solution has the form

$$
g_{0 n}(r, \zeta)=c_{1} J_{n}(\lambda r)+c_{2} Y_{n}(\lambda r)
$$

The constants of integration $c_{1}$ and $c_{2}$ are determined by taking into account (4) in boundary conditions.

The function $g_{1 n}$ occurring in equation (4), is a solution of the same homogeneous differential equation and satisfies the following conditions [2]

$$
\left.g_{1 n}(r, \zeta)\right|_{r=\zeta}=0,\left.\quad \frac{\partial}{\partial r} g_{1 n}(r, \zeta)\right|_{r=\zeta}=\frac{1}{\zeta}
$$


Using the function (5) in conditions (6), after transformation, one obtains

$$
g_{1 n}(r, \zeta)=\frac{\pi}{2}\left[J_{n}(\lambda \zeta) Y_{n}(\lambda r)-J_{n}(\lambda r) Y_{n}(\lambda \zeta)\right]
$$

\section{Circular region with Neumann boundary condition}

For the circular region the Neumann boundary condition is

$$
\left.\frac{\partial G_{n}(r, \zeta)}{\partial r}\right|_{r=b}=0
$$

In this case we have $c_{2}=0$ in equation (5) because we assume that $\left|G_{n}(0, \zeta)\right|<\infty$. Taken into account the boundary conditions $(8)$, we obtain the function $g_{0 n}$ in the form

$$
g_{0 n}(r, \zeta)=\frac{\pi}{2}\left[Y_{n}(\lambda \zeta)-\frac{p_{n}(b)}{q_{n}(b)} J_{n}(\lambda \zeta)\right] J_{n}(\lambda r)
$$

where: $p_{n}(r)=Y_{n+1}(\lambda r)-Y_{n-1}(\lambda r), q_{n}(r)=J_{n+1}(\lambda r)-J_{n-1}(\lambda r)$.

Finally, using equations (4), (7) and (9), the Green's function $G_{n}(r, \zeta)$ for circular region with the Neumann boundary condition at $r=b$, it can written as (Fig. 1a)

$$
G_{n}(r, \zeta)= \begin{cases}\frac{\pi}{2}\left[Y_{n}(\lambda \zeta)-\frac{p_{n}(b)}{q_{n}(b)} J_{n}(\lambda \zeta)\right] J_{n}(\lambda r) & \text { for } 0 \leq r<\zeta \leq b \\ \frac{\pi}{2}\left[Y_{n}(\lambda r)-\frac{p_{n}(b)}{q_{n}(b)} J_{n}(\lambda r)\right] J_{n}(\lambda \zeta) & \text { for } 0 \leq \zeta \leq r \leq b\end{cases}
$$

\section{Annular region with Dirichlet-Neumann boundary conditions}

The Dirichlet and Neumann conditions at boundary of an annular region: $r=a$ and $r=b$, respectively, are

$$
\left.G_{n}(r, \zeta)\right|_{r=a}=0,\left.\frac{\partial}{\partial r} G_{n}(r, \zeta)\right|_{r=b}=0, a \leq r \leq b
$$

The Green's function is defined by equation (4) with the function $g_{1 n}$ given by equation (7). The function $g_{0 n}$ is determined by using the boundary conditions (11). This function is as follows 


$$
g_{0 n}(r, \zeta)=\frac{\pi\left[Y_{n}(\lambda \zeta) q_{n}(b)-J_{n}(\lambda \zeta) p_{n}(b)\right]\left[Y_{n}(\lambda a) J_{n}(\lambda r)-J_{n}(\lambda a) Y_{n}(\lambda r)\right]}{2\left[Y_{n}(\lambda a) q_{n}(b)-J_{n}(\lambda a) p_{n}(b)\right]}
$$

The Green's function $G_{n}(r, \zeta)$ for the annular region with Dirichlet-Neumann boundary conditions using equations (4), (7) and (12) we obtain in the form (Fig. 1b)

$$
G_{n}(r, \zeta)= \begin{cases}\frac{\pi}{2} \frac{\phi_{n}(\zeta, b)}{\phi_{n}(a, b)}\left[Y_{n}(\lambda a) J_{n}(\lambda r)-J_{n}(\lambda a) Y_{n}(\lambda r)\right] & \text { for } a \leq r<\zeta \leq b \\ \frac{\pi}{2} \frac{\phi_{n}(r, b)}{\phi_{n}(a, b)}\left[Y_{n}(\lambda a) J_{n}(\lambda \zeta)-J_{n}(\lambda a) Y_{n}(\lambda \zeta)\right] & \text { for } a \leq \zeta \leq r \leq b\end{cases}
$$

where $\phi_{n}(\alpha, \beta)=Y_{n}(\lambda \alpha) q_{n}(\beta)-J_{n}(\lambda \alpha) p_{n}(\beta)$.

\section{Annular region with Neumann-Neumann boundary conditions}

The Green's function $G_{n}(r, \zeta)$ for the Helmholtz equation with Neumann-Neumann conditions is given by equations (4) and (7). The boundary conditions are:

$$
\left.\frac{\partial}{\partial r} G_{n}(r, \zeta)\right|_{r=a}=\left.\frac{\partial}{\partial r} G_{n}(r, \zeta)\right|_{r=b}=0, a \leq r \leq b
$$

The function $g_{0 n}$ obtained by using the boundary conditions (14) has the form:

$$
g_{0 n}(r, \zeta)=\frac{\pi\left[Y_{n}(\lambda \zeta) q_{n}(b)-J_{n}(\lambda \zeta) p_{n}(b)\right]\left[Y_{n}(\lambda r) q_{n}(a)-J_{n}(\lambda r) p_{n}(a)\right]}{2\left[p_{n}(b) q_{n}(a)-p_{n}(a) q_{n}(b)\right]}
$$

and the Green's function corresponding to these conditions is (Fig. 1c)

$$
G_{n}(r, \zeta)= \begin{cases}\frac{\pi}{2} \frac{\phi_{n}(\zeta, b) \phi_{n}(r, a)}{p_{n}(b) q_{n}(a)-p_{n}(a) q_{n}(b)} & \text { for } a \leq r<\zeta \leq b \\ \frac{\pi}{2} \frac{\phi_{n}(r, b) \phi_{n}(\zeta, a)}{p_{n}(b) q_{n}(a)-p_{n}(a) q_{n}(b)} & \text { for } a \leq \zeta \leq r \leq b\end{cases}
$$




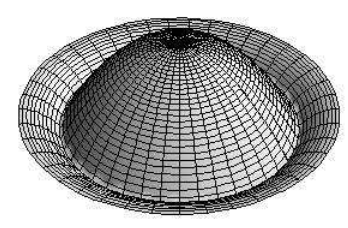

(a)

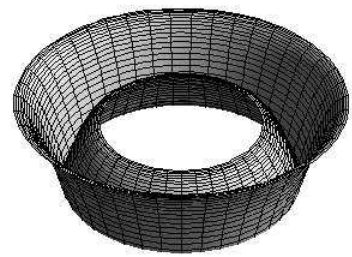

(c)

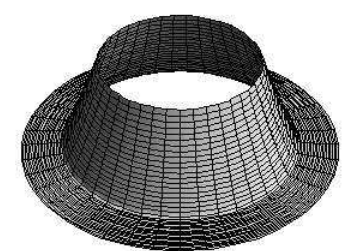

(b)

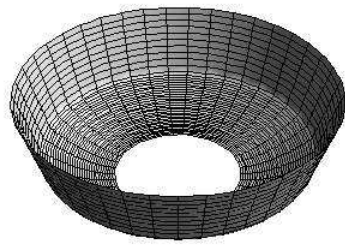

(d)

Fig. 1. The Green's functions for: a) the circular region with Neumann boundary condition,

b) the annular region with Dirichlet-Neumann boundary conditions, c) the annular region with Neumann-Neumann boundary conditions, d) the annular region with Neumann-Dirichlet boundary conditions, $n=0, \Omega=2$

\section{Annular region with Neumann-Dirichlet boundary conditions}

For boundary conditions:

$$
\left.\frac{\partial}{\partial r} G_{n}(r, \zeta)\right|_{r=a}=0,\left.\quad G_{n}(r, \zeta)\right|_{r=b}=0, a \leq r \leq b
$$

the function $g_{0 n}$ has the form:

$$
g_{0 n}(r, \zeta)=\frac{\pi\left[J_{n}(\lambda \zeta) Y_{n}(\lambda b)-J_{n}(\lambda b) Y_{n}(\lambda \zeta)\right]\left[Y_{n}(\lambda r) q_{n}(a)-J_{n}(\lambda r) p_{n}(a)\right]}{-2\left[Y_{n}(\lambda b) q_{n}(a)-J_{n}(\lambda b) p_{n}(a)\right]}
$$

and the Green's function is (Fig. 1d)

$$
G_{n}(r, \zeta)= \begin{cases}-\frac{\pi}{2} \frac{\phi_{n}(r, a)}{\phi_{n}(b, a)}\left[J_{n}(\lambda \zeta) Y_{n}(\lambda b)-J_{n}(\lambda b) Y_{n}(\lambda \zeta)\right] & \text { for } a \leq r<\zeta \leq b \\ -\frac{\pi}{2} \frac{\phi_{n}(\zeta, a)}{\phi_{n}(b, a)}\left[J_{n}(\lambda r) Y_{n}(\lambda b)-J_{n}(\lambda b) Y_{n}(\lambda r)\right] & \text { for } a \leq \zeta \leq r \leq b\end{cases}
$$




\section{Green's functions for exterior Helmholtz problems}

The solution of the Helmholtz problem in a bounded domain can be obtained by using the free space Green's function in the method of fundamental solutions $[4,5]$. The function for problems in $2 \mathrm{D}$ domain has the form:

$$
G_{n}(P, Q)=\frac{i}{4} H_{n}^{(1)}(\lambda d(P, Q))
$$

where $H_{0}^{(1)}(\cdot)$ is the Hankel function of the first kind and zero order, $d(P, Q)=\sqrt{(x-\xi)^{2}+(y-\eta)^{2}}$ where $P=(x, y), Q=(\xi, \eta), \quad i=\sqrt{-1}$. In polar coordinates we have: $x=r \cos \varphi, y=r \sin \varphi, \quad \xi=\rho \cos \vartheta, y=\rho \sin \vartheta \quad$ and $d(P, Q)=\sqrt{r^{2}+\rho^{2}-2 r \rho \cos (\varphi-\vartheta)}$.

We present below the free space Green's function for Helmholtz equation considered in the infinite region $D$ exterior to a centered circle with radius $a$. We assume at the boundary $\partial D$ the Dirichlet or the Neumann condition. These functions can be derived by the use of separation of variables as was shown in the chapter 1 for interior Green's functions. In this case the one-dimensional free space Green's function can be determined as a solution of equation (3) for $r>a$.

The general solution of the problem has the form given by equation (5). The function $g_{1 n}(r, \zeta)$ is also the same as in interior problems and is written by equation (7).

The Dirichlet boundary condition at $r=a$ leads to the relationship

$$
c_{2}=-\frac{J_{n}(\lambda a)}{Y_{n}(\lambda a)} c_{1}
$$

Therefore, the free space Green's function for exterior Helmholtz problems has the form (Fig. 2a):

$$
G_{n}(r, \zeta)=c_{1}\left[J_{n}(\lambda r)-\frac{J_{n}(\lambda a) Y_{n}(\lambda r)}{Y_{n}(\lambda a)}\right]+g_{1 n}(r, \zeta) H(r-\zeta)
$$

The constant $c_{1}$ can be determined by using a condition at infinity. Using the free space Green's function in the method of fundamental solutions the constant can be assumed as an arbitrary value.

Similarly, for Neumann condition at $r=a$ we obtain

$$
c_{2}=-\frac{J_{n-1}(\lambda a)-J_{n+1}(\lambda a)}{Y_{n-1}(\lambda a)-Y_{n+1}(\lambda a)} c_{1}
$$


and the free space Green's function can be presented in the form (Fig. 2b):

$G_{n}(r, \zeta)=c_{1}\left[J_{n}(\lambda r)-\frac{J_{n-1}(\lambda a)-J_{n+1}(\lambda a)}{Y_{n-1}(\lambda a)-Y_{n+1}(\lambda a)} Y_{n}(\lambda r)\right]+g_{1 n}(r, \zeta) H(r-\zeta)$
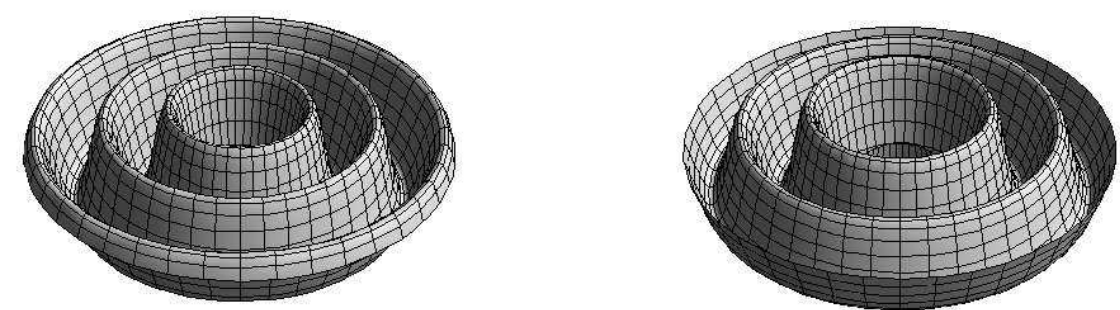

Fig. 2. The Green's functions for exterior Helmholtz problems (a) with the Dirichlet boundary condition, (b) with the Neumann boundary condition, $n=0, \Omega=2$

\section{Example of application}

Let us consider the vibration of a circular membrane consisting with circular and annular regions presented in Figure 3. The transverse vibration of the membrane segments are governed by differential equations

$$
s\left(\frac{\partial^{2} u_{k}}{\partial r^{2}}+\frac{1}{r} \frac{\partial u_{k}}{\partial r}+\frac{1}{r^{2}} \frac{\partial^{2} u_{k}}{\partial \theta^{2}}\right)-\rho_{k} \frac{\partial^{2} u_{k}}{\partial t^{2}}=0, k=1,2
$$

where $s$ is the tension per unit length, $\rho_{k}$ is the density of $k$-th segment of the membrane, $r, \theta$ are polar coordinates and $t$ is time.

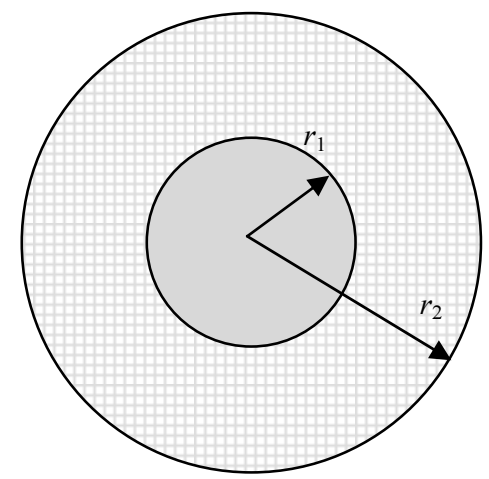

Fig. 3. A sketch of a circular membrane consisting with circular and annular regions 
The functions $u_{k}$ satisfy the continuity conditions at $r=r_{1}$

$$
\begin{gathered}
u_{1}\left(r_{1}, \theta, t\right)=u_{2}\left(r_{1}, \theta, t\right) \\
\left.\frac{\partial u_{1}(r, \theta, t)}{\partial r}\right|_{r=r_{1}}=\left.\frac{\partial u_{2}(r, \theta, t)}{\partial r}\right|_{r=r_{1}}
\end{gathered}
$$

and boundary condition:

$$
u_{2}\left(r_{2}, \theta, t\right)=0
$$

Assuming the functions $u_{k}$ in (25)-(28) in the form

$$
u_{k}(r, \theta, t)=U_{k n}(r) \cos n \theta \cos \omega t
$$

we obtain the following boundary-value problem:

$$
\begin{gathered}
\left\{\begin{array}{c}
\frac{1}{r} \frac{d}{d r}\left(r \frac{d U_{1 n}(r)}{d r}\right)+\left(\lambda_{1}^{2}-\frac{n^{2}}{r^{2}}\right) U_{1 n}(r)=0 \\
\frac{1}{r} \frac{d}{d r}\left(r \frac{d U_{2 n}(r)}{d r}\right)+\left(\lambda_{2}^{2}-\frac{n^{2}}{r^{2}}\right) U_{2 n}(r)=0
\end{array}\right. \\
U_{1 n}\left(r_{1}\right)=U_{2 n}\left(r_{1}\right),\left.\quad \frac{d U_{1 n}(r)}{d r}\right|_{r=r_{1}}=\left.\frac{d U_{2 n}(r)}{d r}\right|_{r=r_{1}} \\
U_{2 n}\left(r_{2}\right)=0
\end{gathered}
$$

where $\lambda_{1}=\omega \sqrt{\frac{\rho_{1}}{s}}, \lambda_{2}=\omega \sqrt{\frac{\rho_{2}}{s}}$.

The solution of the problem (30)-(32) as is shown in paper [6] may be presented with the use of the Green's functions for the Helmholtz problem in the circular region with Neumann boundary condition (4), (7), (9) and in the annular region with Neumann-Dirichlet boundary conditions (4), (7), (16)

$$
\begin{array}{ll}
U_{1 n}(r)=S_{1 n} G_{1 n}\left(r, r_{1}\right), & 0 \leq r \leq r_{1} \\
U_{2 n}(r)=-S_{1 n} G_{2 n}\left(r, r_{1}\right), & r_{1}<r \leq r_{2}
\end{array}
$$

Functions $U_{1 n}, U_{2 n}$ satisfy the conditions (31b), (32). Taking into account equations (33) in condition (31a) we obtain 


$$
S_{1 n}\left[G_{1 n}\left(r_{1}, r_{1}\right)+G_{2 n}\left(r_{1}, r_{1}\right)\right]=0
$$

This equation is fulfilled when

$$
G_{1 n}\left(r_{1}, r_{1}\right)+G_{2 n}\left(r_{1}, r_{1}\right)=0
$$

Equation (35) is the frequency equation which is solved numerically with respect to $\omega_{n}$.

Numerical results of the non-dimensional free vibration frequencies $\Omega_{n}=\omega_{n} r_{2} \sqrt{\frac{\rho_{2}}{s}}$ for the various values of $\sigma=\frac{\rho_{1}}{\rho_{2}}$ are presented in Table 1.

Table 1

Frequency parameter values $\Omega_{0 k}$ of the composite circular membrane shown in Figure 3 for various values of $\sigma=\rho_{1} / \rho_{2}\left(r_{1} / r_{2}=0.5\right)$

\begin{tabular}{|c|c|c|c|c|c|}
\hline$\sigma$ & $\Omega_{01}$ & $\Omega_{02}$ & $\Omega_{03}$ & $\Omega_{04}$ & $\Omega_{05}$ \\
\hline 0.1 & 3.57325 & 9.55645 & 15.73338 & 21.94047 & 28.14370 \\
\hline 1.0 & 2.40483 & 5.52008 & 8.65373 & 11.79153 & 14.93092 \\
\hline 5.0 & 0.57109 & 1.64999 & 2.84236 & 4.04593 & 5.20686 \\
\hline 10.0 & 0.28701 & 0.83304 & 1.43825 & 2.05526 & 2.67589 \\
\hline
\end{tabular}

\section{Conclusions}

The Green's functions which are used in solving the Helmholtz equation occurring in vibration problems of composite membranes have been derived. The closed form of the Green's function is obtained as a solution of the boundary problem for circular and annular domains for various boundary conditions. For the exterior Helmholtz problem the free space Green's functions have been presented. These functions can be used in the method of fundamental solutions applying to Helmholtz equation in domains with a circular hole. The presented example shows the use of the Green's functions in vibration analysis of a composite membrane consisting in circular and annular regions.

\section{References}

[1] Duffy D.G., Green's functions with applications, Chapman \& Hall/CRC, Boca Raton, Washington 2001.

[2] Kukla S., Funkcje Greena i ich zastosowania, Seria Monografie Nr 170, Wydawnictwo Politechniki Częstochowskiej, Częstochowa 2009. 
[3] Pérez-Arancibia C., Durán M., On the Green's function for the Helmholtz operator in an impedance circular cylindrical waveguide, Journal of Computational and Applied Mathematics 2010, 235, 244-262.

[4] Bin-Mohsin B., Lesnic D., The method of fundamental solutions for Helmholtz-type equations in composite materials, Computers and Mathematics with Applications 2011, 62, 4377-4390.

[5] Reutskiy S.Yu., The method of fundamental solutions for Helmholtz eigenvalue problems in simply and multiply connected domains, Engineering Analysis with Boundary Elements 2006, 30, 150-159.

[6] Siedlecka U., Kukla S., Zamorska I., Free vibration of composite circular membranes, Scientific Research of the Institute of Mathematics and Computer Science Czestochowa University of Technology 2012, 1(11), 96-106.

[5] Zamorska I., Kukla S., Siedlecka U., Frequency analysis of composite annular membranes, Scientific Research of the Institute of Mathematics and Computer Science Czestochowa University of Technology 2012, 1(11), 131-137. 